\title{
ARISTOLOCHIA RZEDOWSKIANA (ARISTOLOCHIACEAE), UNA ESPECIE NUEVA PARA LA SUBSECCIÓN PENTANDRAE DEL ESTADO DE JALISCO, MÉXICO
}

\author{
Francisco J. Santana-Michel ${ }^{1,2}$ y Luis Guzmán-HernándeZ ${ }^{1}$ \\ ${ }^{1}$ Universidad de Guadalajara, Centro Universitario de la Costa Sur, \\ Departamento de Ecología y Recursos Naturales, Independencia Nacional \\ Núm. 151, Apdo. postal 64, 48900 Autlán, Jalisco, México. \\ ${ }^{2}$ Autor para la correspondencia: fsantanam@cucsur.udg.mx
}

\section{RESUMEN}

Aristolochia rzedowskiana Santana-Michel \& Guzmán-Hernández es descrita como especie nueva de una localidad cercana a San Sebastián del Oeste y Puerto Vallarta, en el estado de Jalisco, México; pertenece a Aristolochia sect. Gymnolobus subsect. Pentandrae. Esta planta habita en bosques tropicales subcaducifolios y encinares; se puede identificar por su limbo del perianto de color rojizo, cordado-lanceolado, reflexo o recto, obtuso o atenuado hacia el ápice, superficie del limbo papiloso-reticulada, garganta pálido-amarillenta con puntos obscuros. Por sus caracteres morfológicos se relaciona con Aristolochia variifolia, A. karwinskii y A. manantlanensis.

Palabras clave: Aristolochia rzedowskiana, Aristolochiaceae, Jalisco, Pentandrae.

\section{ABSTRACT}

Aristolochia rzedowskiana Santana-Michel \& Guzmán-Hernández is described as a new species from a locality near San Sebastián del Oeste and Puerto Vallarta in the State of Jalisco, Mexico; it belongs to Aristolochia sect. Gymnolobus subsect. Pentandrae. This species inhabits tropical subdeciduous forests and oak woods and can be identified by its perianth limb which is reddish, cordate-lanceolate, reflexed or straight, obtuse or attenuate towards the apex, with a papillose-reticulate surface and pale yellowish throat with dark spots. Its morphological characters relate it to Aristolochia variifolia, A. karwinskii and A. manantlanensis.

Key words: Aristolochia rzedowskiana, Aristolochiaceae, Jalisco, Pentandrae. 
Las especies del género Aristolochia L. pertenecientes a la sección Gymnolobus Duch. subsect. Pentandrae Duch. se caracterizan por presentar cinco estambres y cinco carpelos. Casi en su totalidad son endémicas de México, solo Aristolochia coryi Johnston crece en Texas en los Estados Unidos de América (E.U.A.), tres comparten su distribución entre México y ese país, y una, A pentandra Jacq., se distribuye en México, Bahamas, Cuba, Jamaica, los E.U.A. y recientemente fue encontrada en la isla San Andrés, Colombia (Pfeifer, 1970; González et. al., 2010). La subsect. Hexandrae, cuyas especies poseen seis estambres y seis carpelos, se distribuye desde el sur de los E.U.A. hasta Suramérica (Pfeifer, 1966). Recientemente, evidencias moleculares y morfológicas sugieren que el grupo hermano de la subsect. Pentandrae es un conjunto de cinco especies hexandras de Suramérica subtropical y templada, lo cual constituye la disyunción biogeográfica entre Centro y Suramérica y la zona templada y subtropical de América del Norte (González et al., 2010).

En 1970 Rogers McVaugh colectó en el estado de Jalisco en el municipio de Cabo Corrientes una planta perteneciente a Aristolochia subsect. Pentandrae que posteriormente fue identificada por Howard Pfeifer como Aristolochia variifolia Duch. Al revisar material procedente de esa región notamos que se trata de una especie diferente, la cual proponemos a continuación como especie nueva.

Aristolochia rzedowskiana Santana-Michel \& Guzmán-Hernández sp. nov., Figs. 1 y 2 .

Aristolochia variifolia simillima perianthio rubello fauce maculis atropurpureis instructa limbo papilloso reticulato differt.

Plantas perennes, procumbentes, de 1-2 m de largo; raíz principal axonomorfa; hojas pecioladas, auriculiformes, obovadas, base bilobada, lóbulos redondeados, agudas a acuminadas en el ápice, haz finamente escabroso, envés esparcidamente piloso, nervaduras principales y el borde pilosos, nervación actinódroma basal; pecíolo piloso de (0.8)1-1.5(2) $\mathrm{cm}$ de largo, 0.7-1 mm de grueso; lámina de (3) 4-8(11) cm de largo, (1.5)3-4(5) cm de ancho; flores de olor fétido, 1-2 flores axilares, pedúnculo bracteolado, de (4)5-8.5(12) $\mathrm{mm}$ de largo y 1-1.5 mm de grueso, bractéolas lanceoladas, pilosas, de (4)5-7(11) $\mathrm{mm}$ de largo, 2-3.5(5) $\mathrm{mm}$ de ancho; perianto de $3.5-5 \mathrm{~cm}$, la parte basal forma con la parte apical un ángulo de $150^{\circ}$, rojizo, la garganta pálido-amarillenta con puntos oscuros, hipantio no evidente, limbo cordiforme, lanceolado, reflexo o recto, más largo que ancho, 5-nervado, 


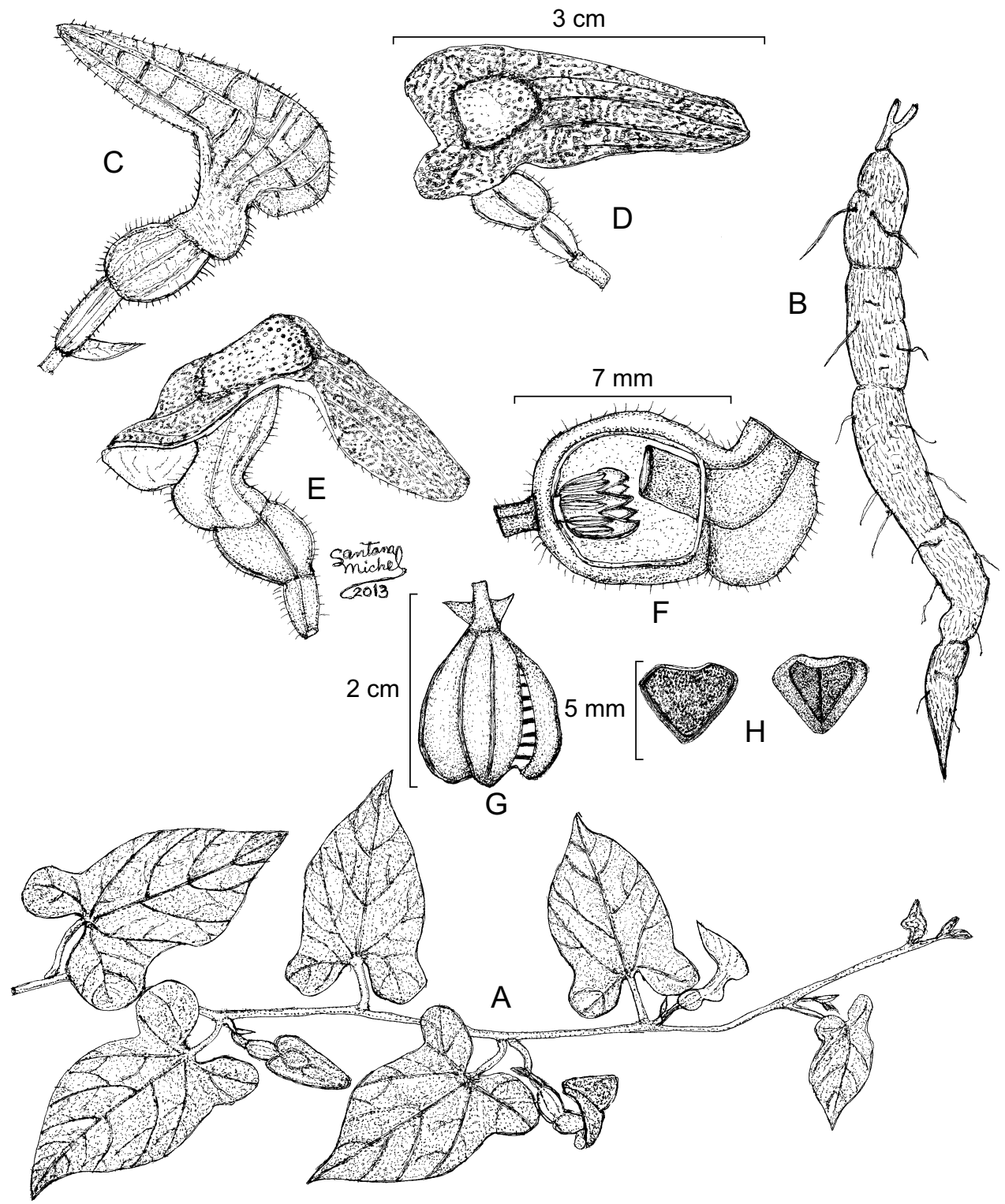

Fig. 1. Aristolochia rzedowskiana. A. aspecto de la planta; B. raíz principal; C. flor cerrada; D. flor vista de frente; E. flor vista de costado; F. corte longitudinal del utrículo, mostrando la siringe y el ginostemo; G. fruto; H. semilla. Ilustración basada en la colección tipo (F. $J$. Santana-Michel y L. Guzmán H. 12366). 

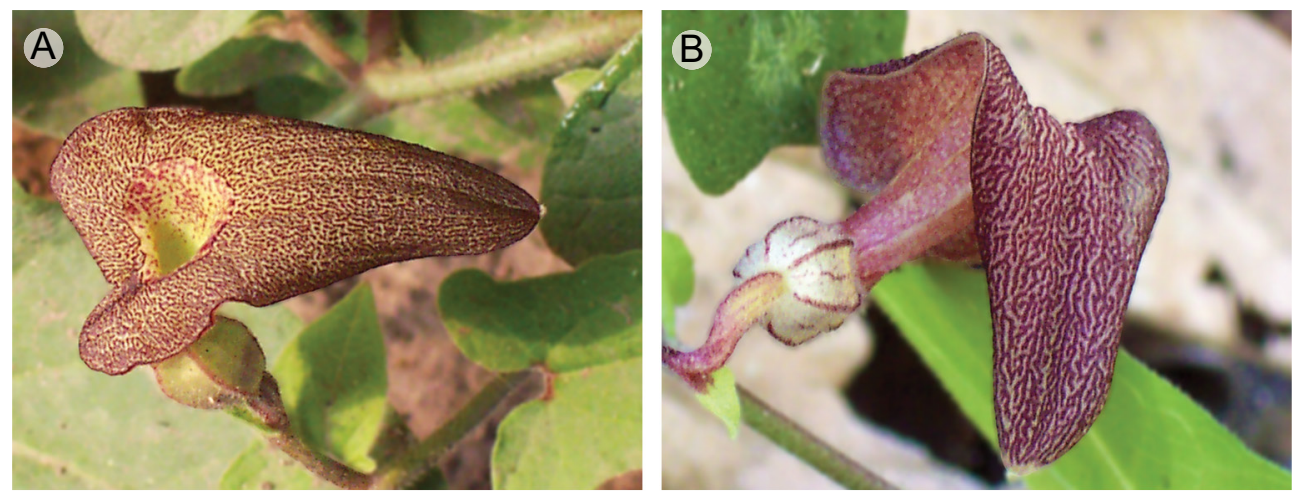

Fig. 2. Flor de Aristolochia rzedowskiana vista de frente (A) y de costado (B).

entero, obtuso o atenuado hacia el ápice, de 2.5-3.5 $\mathrm{cm}$ de largo, $1-1.5 \mathrm{~cm}$ de ancho, rojizo, papiloso-reticulado, excepto en la garganta, tubo doblado $80-90^{\circ}$, piloso en los nervios reticulados purpúreos, con una protuberancia en la parte basal junto al utrículo, de color verde a purpúreo, de (1.0)1-1.5(2) $\mathrm{cm}$ de largo, 2.5-3.5 $\mathrm{mm}$ de diámetro, utrículo oblongo-elipsoide, de 5-8 $\mathrm{mm}$ de largo, 4-6 $\mathrm{mm}$ de diámetro, siringe excéntrica, tubular, de 2-3.3 mm de largo, 1.5-1.8 mm de diámetro; ginostemo 5-lobado, subestipitado, de 1.5-2.5 $\mathrm{mm}$ largo, 1.5-2 $\mathrm{mm}$ de diámetro, estípite de $0.6-1 \mathrm{~mm}$ de largo, 0.5-1 $\mathrm{mm}$ de diámetro, estambres 5 , tetraloculares, anteras de 1-1.4 $\mathrm{mm}$ de largo, 0.4-0.6 $\mathrm{mm}$ de ancho; ovario piloso, de 5-7(8) $\mathrm{mm}$ de largo, 1.5-2 $\mathrm{mm}$ de diámetro; fruto capsular, subgloboso, septífraga marginal basípeta, de $1.8-2.5 \mathrm{~cm}$ de largo, $1.8-2.0 \mathrm{~cm}$ de diámetro; semillas numerosas (40-50 por fruto), triangulares, de color café, de 4-5 $\mathrm{mm}$ de largo, 4-6 mm de ancho, $1 \mathrm{~mm}$ de grueso, la superficie ligeramente tuberculada.

Tipo: MÉXICO. Jalisco, municipio de Puerto Vallarta, Potrero de Abajo, entre La Estancia y Las Palmas de Arriba, por la carretera Mascota - Puerto Vallarta, altitud $360 \mathrm{~m}$, bosque tropical subcaducifolio con Brosimum, Hura, Enterolobium, Ficus, Acacia y Sapium, 9 de diciembre de 2006, F.J. Santana-Michel y L. GuzmánHernández 12366 (holótipo en ZEA; isótipos por distribuirse a los herbarios ENCB, IBUG, IEB y MEXU).

Material adicional examinado: MÉXICO. Jalisco, municipio de Cabo Corrientes, $5 \mathrm{~km}$ North of El Tuito, 16-17 de diciembre de 1970, R. McVaugh 25460 (MICH); 
municipio de Puerto Vallarta, Arroyo de Las Palmas, $3 \mathrm{~km}$ north of Las Palmas, 19 de diciembre de 1970, R. McVaugh 25570 (MICH); entre La Estancia y Las Palmas de Arriba, por la carretera Mascota - Puerto Vallarta, 400 m s.n.m., bosque tropical subcaducifolio con Brosimum, Enterolobium, Hura, Acacia y Sapium, 9 de diciembre de 2006, F.J. Santana-Michel y L. Guzmán Hernández 12355 (ZEA); Potrero de Abajo, entre La Estancia y Las Palmas de Arriba, sobre el Río Las Palmas, por la carretera Mascota - Puerto Vallarta, 400 m s.n.m., bosque tropical subcaducifolio con Brosimum, Hura, Enterolobium, Ficus, Acacia, Cymbopetalum y Luehea, 9 de diciembre de 2006, F.J. Santana-Michel y L. Guzmán Hernández 12359 (ZEA); La Capulinosa, $2 \mathrm{~km}$ al SE de Las Palmas de Arriba, $200 \mathrm{~m}$, bosque tropical subcaducifolio con Brosimum, Ficus, Hura, Randia, Acacia, 9 de diciembre de 2006, F.J. Santana-Michel y L. Guzmán Hernández 12368 (ZEA); Los Arrayanes, entre La Estancia y Las Palmas de Arriba, por la carretera Mascota - Puerto Vallarta, $271 \mathrm{~m}$, bosque tropical subcaducifolio con Brosimum, Astronium, Ficus, Cecropia, Acacia, Cymbopetalum and Couepia, 22 de noviembre de 2009, F.J. Santana-Michel, L. Guzmán-Hernández y A. Paizanni Guillén 14072 (ZEA); municipio San Sebastián del Oeste, $9 \mathrm{~km}$ (en línea recta) y $14.9 \mathrm{~km}$ (por la brecha a Las Palmas) al NO de La Estancia, 500-600 m s.n.m., bosque de encino con Byrsonima crassifolia, 28 de julio de 2004, P. Carrillo-Reyes y F. Nicolalde 4296 (IBUG, ZEA); municipio de Talpa de Allende, $16-17 \mathrm{~km}$ al SSE de Talpa por el camino a La Cuesta, $1370 \mathrm{~m}$ s.n.m., bosque de coníferas con elementos de mesófilo, 22 de agosto de 2004, P. Carrillo-Reyes y A. Kennedy 4391 (IEB, ZEA).

Distribución conocida: en los municipios de Cabo Corrientes, Puerto Vallarta, San Sebastián del Oeste y Talpa de Allende del estado de Jalisco, México.

Época de floración: de agosto a diciembre y quizás hasta enero.

Hábitat: bosque tropical subcaducifolio con las especies arbóreas predominantes: Brosimum alicastrum, Astronium graveolens, Ficus insipida, Cecropia obtusifolia, Couepia polyandra, Acacia polyphylla, Cymbopetalum hintonii, Enterolobium cyclocarpum, Hura polyandra, Sapium macrocarpum, Randia armata, Bursera simaruba, Luehea candida, también en bosque de encino con Quercus magnoliifolia, $Q$. resinosa, $Q$. castanea, $Q$. gentryi, $Q$. elliptica y Lysiloma acapulcense.

Etimología: el epíteto específico se dedica en honor al Dr. Jerzy Rzedowski Rotter, como reconocimiento por sus aportaciones a la botánica en México. 
De acuerdo con Pfeifer (1970), Aristolochia rzedowskiana se ubica junto a $A$. variifolia Duch., A. karwinskii Duch. y A. manantlanensis Santana-Michel, especies con flores geniculadas y periantos maduros mayores de $3 \mathrm{~cm}$ de largo, pedúnculos cortos y el tubo del cáliz doblado a más de $90^{\circ}$. Aristolochia manantlanensis difiere de A. rzedowskiana por sus láminas sagitadas con la base bilobada, pecíolos de $2.5-5 \mathrm{~cm}$ de largo, bractéolas triangular cordadas, limbo del perianto ovado peltado, recto, de color púrpura obscuro, superficie del limbo piloso-fimbriado excepto en la garganta; tubo tubular; utrículo elipsoide; garganta glabra, amarilla (Cuadro 1).

Cuadro 1. Comparación de caracteres de A. rzedowskiana con las otras especies relacionadas.

\begin{tabular}{|c|c|c|c|c|}
\hline & A. variifolia & A. karwinskii & A. manantlanensis & A. rzedowskiana \\
\hline Lámina foliar & $\begin{array}{l}\text { cordada } \\
\text { a hastada } \\
\text { subtrilobada }\end{array}$ & $\begin{array}{l}\text { triangular- } \\
\text { cordada } \\
\text { a hastada } \\
\text { subtrilobada }\end{array}$ & $\begin{array}{l}\text { sagitada, con la } \\
\text { base bilobada }\end{array}$ & $\begin{array}{l}\text { obovada } \\
\text { auriculiforme }\end{array}$ \\
\hline Pecíolos & $1-2 \mathrm{~cm}$ de largo & $1-3 \mathrm{~cm}$ de largo & $2-5 \mathrm{~cm}$ de largo & $\begin{array}{l}(0.8) 1-1.5(2) \mathrm{cm} \\
\text { de largo }\end{array}$ \\
\hline \multicolumn{5}{|c|}{ Limbo del perianto } \\
\hline forma & $\begin{array}{l}\text { ovado-cordado, } \\
\text { recto, agudo en } \\
\text { el ápice }\end{array}$ & $\begin{array}{l}\text { ovado, recto, } \\
\text { agudo en el } \\
\text { ápice }\end{array}$ & $\begin{array}{l}\text { ovado-peltado, } \\
\text { recto, obtuso en el } \\
\text { ápice }\end{array}$ & $\begin{array}{l}\text { cordiforme, } \\
\text { lanceolado, } \\
\text { reflexo o recto, } \\
\text { obtuso o agudo } \\
\text { en el ápice }\end{array}$ \\
\hline superficie & $\begin{array}{l}\text { cortamente } \\
\text { fimbriada }\end{array}$ & glabrescente & piloso-fimbriada & $\begin{array}{l}\text { papiloso- } \\
\text { reticulada }\end{array}$ \\
\hline color & café & púrpura oscuro & $\begin{array}{l}\text { púrpura oscuro } \\
\text { con una línea } \\
\text { verde amarillenta }\end{array}$ & rojizo \\
\hline tubo & $\begin{array}{l}\text { agrandándose } \\
\text { hacia el limbo }\end{array}$ & tubular & tubular & $\begin{array}{l}\text { con una } \\
\text { protuberancia } \\
\text { en la base }\end{array}$ \\
\hline garganta & pálida & café & amarilla & $\begin{array}{l}\text { pálido- } \\
\text { amarillenta con } \\
\text { puntos oscuros }\end{array}$ \\
\hline Utrículo & elipsoide & obcónico & elipsoide & $\begin{array}{l}\text { oblongo- } \\
\text { elipsoide }\end{array}$ \\
\hline
\end{tabular}


Las cuatro especies relacionadas se pueden separar mediante la siguiente clave:

1 Limbo del perianto café o púrpura con la superficie cortamente fimbriada a glabrescente.

2 Superficie del limbo del perianto glabrescente, garganta con la superficie periférica más oscura A. karwinskii

2 Superficie del limbo del perianto cortamente fimbriada o piloso-fimbriada garganta pálida o amarilla, glabra.

3 Limbo del perianto de $2.5 \mathrm{~cm}$ de largo, $1.5 \mathrm{~cm}$ de ancho, ápice del limbo agudo, garganta pálida ...................................................... . variifolia

3 Limbo del perianto de 2.4-2.8 cm de largo, 1.7-2.4 cm de ancho, ápice del limbo obtuso, garganta amarilla ................................ A. manantlanensis

1 Limbo del perianto rojizo con la superficie papiloso-reticulada .. A. rzedowskiana

\section{AGRADECIMIENTOS}

Los autores desean agradecer a las autoridades del Centro Universitario de la Costa Sur de la Universidad de Guadalajara y especialmente al Departamento de Ecología y Recursos Naturales. A Roberto González Tamayo y Luz María González Villarreal por su ayuda en la diagnosis. A Pablo Carrillo Reyes por facilitarnos material de esta especie. Asimismo a nuestros compañeros y colaboradores en el inventario de la región Costa Sur de Jalisco, Ramón Cuevas G., Nora Minerva Núñez L., Enrique V. Sánchez Rodríguez, Arturo Solís Magallanes y Carlos Palomera.

\section{LITERATURA CITADA}

González, F., H. E. Esquivel, G. A. Murcia y N. Pavón-Mora. 2010. Aristolochia pentandra (Aristolochiaceae) in Colombia: biogeographic implications and proposed synapomorphies between the pentandrous species of Aristolochia and its South American sister group. Rev. Acad. Colomb. Cienc. 34(133): 467-478.

Pfeifer, H. W. 1966. Revision of the North and Central American hexandrous species of Aristolochia (Aristolochiaceae). Ann. Missouri Bot. Gard. 53: 115-196.

Pfeifer, H. W. 1970. A revision of the pentandrous species of Aristolochia. The University of Connecticut Publication Series. Storrs, USA. 134 pp. 